\title{
HYPERGEODESIC CURVATURE AND TORSION
}

\section{DAVID B. DEKKER}

1. Introduction. A hypergeodesic curve ${ }^{1}$ on a surface satisfies an ordinary second-order differential equation similar to that for the geodesic curves except that the coefficients, instead of being Christoffel symbols, are taken to be arbitrary functions of the surface coordinates. At a point of the surface the envelope of all the osculating planes of hypergeodesic curves of a family determined by such an equation which pass through the point will be a cone usually of order four and class three with its vertex at the point. If the cone at each point degenerates into a single line of a congruence of lines, then the family of hypergeodesics is the family of union curves $^{2}$ relative to this congruence. Recently, a union curvature ${ }^{3}$ and a union torsion ${ }^{4}$ have been defined relative to a family of union curves as generalizations of geodesic curvature and geodesic torsion.

The following investigation deals with the differential equation for an arbitrary family of hypergeodesic curves on a surface and the definition of a hypergeodesic curvature and a hypergeodesic torsion relative to a family of hypergeodesic curves in such a manner that the definitions will reduce to those for union curvature and union torsion when the family is taken to be a family of union curves. A geometric interpretation of hypergeodesic curvature is given which generalizes the geometric interpretation for union curvature and geodesic curvature. Finally, a geometric condition that a hypergeodesic be a plane curve is obtained as a generalization of a theorem known for union curves.

The notation of Eisenhart ${ }^{5}$ is used throughout except that $\Gamma_{\beta \gamma}^{\alpha}$ is used for Christoffel symbols of the second kind with respect to the coefficients of the first fundamental form for the surface. The summation convention of tensor analysis with regard to repeated indices

Presented to the Society, April 30, 1949; received by the editors September 16, 1948.

${ }^{1}$ E. P. Lane, Projective differential geometry, University of Chicago Press, 1942, p. 192.

2 P. Sperry, Properties of a certain projectively defined two-parameter family of curves on a general surface, Amer. J. Math. vol. 40 (1918) p. 213.

3 C. E. Springer, Union curves and union curvature, Bull. Amer. Math. Soc. vol. 51 (1945) pp. 686-691.

${ }^{4}$ C. E. Springer, Union torsion of a curve on a surface, Amer. Math. Monthly vol. 54 (1947) pp. 259-262.

' L. P. Eisenhart, Differential geometry, Princeton University Press, 1940. 
will be observed with Greek letter indices taking on the values 1, 2 and the Latin letter indices taking on the values 1, 2, 3 .

2. The differential equation of a family of hypergeodesics. Consider a surface $S$ in ordinary space of three dimensions represented by the three equations $x^{i}=x^{i}\left(u^{1}, u^{2}\right)(i=1,2,3)$ referred to a rectangular cartesian coordinate system. The functions $x^{i}$ and their partial derivatives of the second order with respect to the parameters $u^{1}$ and $u^{2}$ are assumed continuous at any point of the surface. The solutions $u^{2}=u^{2}\left(u^{1}\right)$ of an ordinary second order differential equation of the form

$$
\frac{d}{d u^{1}}\left(\frac{d u^{2}}{d u^{1}}\right)=A+B \frac{d u^{2}}{d u^{1}}+C\left(\frac{d u^{2}}{d u^{1}}\right)^{2}+D\left(\frac{d u^{2}}{d u^{1}}\right)^{3},
$$

in which $A, B, C$, and $D$ are analytic functions of $u^{1}$ and $u^{2}$, are the curves of a two-parameter family of hypergeodesics. If $u^{\alpha}=u^{\alpha}(s)$ $(\alpha=1,2)$, where the parameter $s$ is arc length, is a hypergeodesic of the family, then equation (2.1) takes the form

$$
e_{\alpha \beta} u^{\prime \alpha} u^{\prime \beta}=A_{\alpha \beta \gamma} u^{\prime \alpha} u^{\prime \beta} u^{\prime \gamma},
$$

in which $e_{12}=1, e_{21}=-1, e_{11}=e_{22}=0$, and

$$
\begin{aligned}
& A_{111}=A, \quad A_{112}+A_{121}+A_{211}=B, \\
& A_{122}+A_{212}+A_{221}=C, \quad A_{222}=D,
\end{aligned}
$$

and the primes indicate differentiation with respect to $s$.

Under a transformation $\bar{u}^{\alpha}=\bar{u}^{\alpha}\left(u^{1}, u^{2}\right)(\alpha=1,2)$ of surface coordinates for which the Jacobian does not vanish, the differential equation

$$
e_{\alpha \beta} \bar{u}^{\prime \alpha} \bar{u}^{\prime \beta}=\bar{A}_{\tau \sigma \rho} \bar{u}^{\prime \tau} \bar{u}^{\prime \sigma} \bar{u}^{\prime \rho}
$$

is obtained in the new coordinates, where

$$
\bar{A}_{\tau \sigma \rho}=\frac{\partial\left(\bar{u}^{1}, \bar{u}^{2}\right)}{\partial\left(u^{1}, u^{2}\right)}\left[A_{\alpha \beta \gamma} \frac{\partial u^{\alpha}}{\partial \bar{u}^{\tau}} \frac{\partial u^{\beta}}{\partial \bar{u}^{\sigma}} \frac{\partial u^{\gamma}}{\partial \bar{u}^{\rho}}-e_{\alpha \beta} \frac{\partial u^{\alpha}}{\partial \bar{u}^{\tau}} \frac{\partial^{2} u^{\beta}}{\partial \bar{u}^{\sigma} \partial \bar{u}^{\rho}}\right]
$$

It is clear, however, that $A_{\alpha \beta \gamma}$ is not in general a tensor.

In order to express the equation (2.2) in tensor form, consider the geodesic curvature vector ${ }^{6}$ whose contravariant components $\rho^{\tau}$ are

$$
\rho^{\tau}=u^{\prime \prime \tau}+\Gamma_{\beta \gamma}^{\tau} u^{\prime \beta} u^{\prime \gamma} .
$$

Substituting the values of $u^{\prime \prime r}$ from (2.5), equation (2.2) becomes

\footnotetext{
${ }^{6}$ Eisenhart, loc. cit. p. 187.
} 


$$
e_{\alpha \tau} u_{\rho}^{\prime \alpha}{ }^{\tau}=\left[A_{\alpha \beta \gamma}+e_{\alpha \tau} \Gamma_{\beta \gamma}^{\tau}\right] u^{\prime \alpha} u^{\prime \beta} u^{\prime \gamma} .
$$

The left-hand member of this equation becomes a scalar if multiplied by $g^{1 / 2}$, where $g$ is the determinant of the coefficients of the first fundamental form, namely,

$$
g=e^{\alpha \beta} g_{1 \alpha} g_{2 \beta}
$$

with

$$
g_{\alpha \beta}=x^{i},{ }_{\alpha} x_{, \beta}^{i}
$$

and

$$
e^{12}=1, \quad e^{21}=-1, \quad e^{11}=e^{22}=0 .
$$

Hence, the differential equation may be written in the tensor form

$$
\epsilon_{\alpha \tau} u^{\prime \alpha} \rho^{\tau}=\Omega_{\alpha \beta \gamma} u^{\prime \alpha} u^{\prime \beta} u^{\prime \gamma},
$$

where $\epsilon_{\alpha \tau}=g^{1 / 2} e_{\alpha \tau}$ and the functions $\Omega_{\alpha \beta \gamma}$ of $u^{1}$ and $u^{2}$ are defined by

$$
\Omega_{\alpha \beta \gamma} \equiv \epsilon_{\alpha \tau} \Gamma_{\beta \gamma}^{\tau}+g^{1 / 2} A_{\alpha \beta \gamma} .
$$

Since the left member of (2.7) is a scalar, so also is the right member. Consequently, the functions $\Omega_{\alpha \beta \gamma}$ are the triply covariant components of a tensor of the third order; that is, under a transformation of surface coordinates,

$$
\bar{\Omega}_{\alpha \beta \gamma}=\Omega_{\tau \sigma \rho} \frac{\partial u^{\tau}}{\partial \bar{u}^{\alpha}} \frac{\partial u^{\sigma}}{\partial \bar{u}^{\beta}} \frac{\partial u^{\rho}}{\partial \bar{u}^{\gamma}},
$$

as may be verified by direct computation. Now equation (2.7) may be written

$$
\epsilon_{\alpha \tau} u^{\prime \alpha} \rho^{\tau}=\Omega,
$$

in which the scalar $\Omega$ is defined by the right-hand member of (2.7).

To specialize the components $\Omega_{\alpha \beta \gamma}$ to be symmetric in each pair of indices actually constitutes no restriction on the family of hypergeodesics to be considered. Employing this symmetry in (2.8) gives

$$
\begin{aligned}
& A_{211}-A_{112}=\Gamma_{11}^{1}+\Gamma_{21}^{2}, \\
& A_{112}-A_{121}=0, \\
& A_{212}-A_{122}=\Gamma_{22}^{2}+\Gamma_{12}^{1}, \\
& A_{212}-A_{221}=0,
\end{aligned}
$$

which with equations (2.3) are found to have the unique solutions 


$$
\begin{aligned}
A_{111} & =A, \\
A_{112}= & A_{121}=\left(B-\Gamma_{11}^{1}-\Gamma_{21}^{2}\right) / 3, \\
A_{211} & =\left(B+2 \Gamma_{11}^{1}+2 \Gamma_{21}^{2}\right) / 3, \\
A_{212}= & A_{221}=\left(C+\Gamma_{22}^{2}+\Gamma_{12}^{1}\right) / 3, \\
A_{122} & =\left(C-2 \Gamma_{22}^{2}-2 \Gamma_{12}^{1}\right) / 3, \\
A_{222} & =D .
\end{aligned}
$$

Hence, a necessary and sufficient condition that $\Omega_{\alpha \beta \gamma}$ be a symmetric tensor in any pair of indices is that the functions $A_{\alpha \beta \gamma}$ be given by the relations (2.11).

3. Hypergeodesic curvature. The differential equation (2.9) is transformed into two differential equations by simply multiplying it through by the expressions $\epsilon^{\delta \mu} g_{\mu \eta} u^{\prime \eta}(\delta=1,2)$, where $\epsilon^{\delta \mu}=e^{\delta \mu} / g^{1 / 2}$. The left-hand members of the equations so obtained reduce to $-\rho^{\delta}$ $(\delta=1,2)$ from the facts $g_{\beta \eta} u^{\prime \beta} u^{\prime \eta}=1, g_{\tau \eta} \rho^{\tau} u^{\prime \eta}=0$, and a subtraction of $g_{\tau \eta} \rho^{r} u^{\prime} u^{\prime \delta}=0(\delta=1,2)$. Hence, a family of hypergeodesics can be represented by the two differential equations

$$
\lambda^{\delta} \equiv \rho^{\delta}+\epsilon^{\delta \mu} g_{\mu \eta} u^{\prime} \eta \Omega=0 \quad(\delta=1,2) .
$$

The left members of equations (3.1) define the contravariant components $\lambda^{\delta}$ of a vector which will be called the hypergeodesic curvature vector of a curve $C$ given by $u^{\alpha}=u^{\alpha}(s)$. If $\lambda^{\delta}$ is a null vector along a curve, then equations (3.1) are satisfied and the curve is a hypergeodesic of the family, and conversely.

It is easily shown ${ }^{7}$ that $\mu^{\delta}$ defined by

$$
\mu^{\delta} \equiv-\epsilon^{\delta \mu} g_{\mu \eta} u^{\prime \eta}
$$$$
(\delta=1,2)
$$

are the contravariant components of a unit vector which makes a right angle with the unit vector $u^{\prime \alpha}$, so that

$$
\epsilon_{\alpha \delta} u^{\prime \alpha} \mu^{\delta}=1 \text {. }
$$

Now, geodesic curvature ${ }^{8} K_{g}$ is defined by

$$
K_{o} \equiv \epsilon_{\alpha \delta} u^{\prime \alpha} \rho^{\delta},
$$

which implies that

$$
\rho^{\delta}=K_{o} \mu^{\delta},
$$

so that (3.1) assumes the form

7 Eisenhart, loc. cit., p. 136.

8 Eisenhart, loc. cit., p. 187. 


$$
\lambda^{\delta} \equiv\left(K_{o}-\Omega\right) \mu^{\delta}=0 .
$$

Therefore the hypergeodesic curvature vector $\lambda^{\delta}$ makes a right angle with the unit vector $u^{\prime \alpha}$.

Hypergeodesic curvature $K_{h}$ will be defined by

$$
K_{h} \equiv \epsilon_{\alpha \delta} u^{\prime \alpha} \lambda^{\delta},
$$

which by (3.6) and (3.3) gives

$$
K_{h} \equiv K_{o}-\Omega
$$

that is, hypergeodesic curvature is geodesic curvature diminished by the scalar $\Omega$. With the definition (3.4) equation (2.9) may be written

$$
K_{0}=\Omega, \text { or } K_{0}-\Omega=0,
$$

so that a family of hypergeodesics is characterized by the property (3.9); namely, the geodesic curvature is equal to the scalar $\Omega$ which is a function of $u^{1}, u^{2}, u^{\prime 1}$, and $u^{\prime 2}$ only. From (3.8) and (3.9) arises the following theorem which is a generalization of a corresponding theorem for geodesics: a necessary and sufficient condition that a curve $u^{\alpha}=u^{\alpha}(s)$ be a hypergeodesic of a family is that the hypergeodesic curvature along the curve be identically zero in $s$.

4. The elements of the cone related to the hypergeodesics through a point. The elements of the cone enveloped by the osculating planes of a family of hypergeodesics through a point $P$ of the surface $S$ are to be determined in tensor form.

If the curve $u^{\alpha}=u^{\alpha}(s)$ is a hypergeodesic on the surface $S$ given by $x^{i}=x^{i}\left(u^{1}, u^{2}\right)$, then the osculating plane of the hypergeodesic at the point $x^{i}\left(u^{1}(s), u^{2}(s)\right)$ is given by the determinantal equation

$$
\epsilon_{i j k}\left(\bar{x}^{i}-x^{i}\right) \frac{d x^{j}}{d s} \frac{d^{2} x^{k}}{d s^{2}}=0,
$$

where the $\bar{x}^{i}$ are the current coordinates. But from

$$
\frac{d x^{j}}{d s}=x_{,{ }_{\sigma}}^{i} u^{\prime \sigma}, \quad \frac{d^{2} x^{k}}{d s^{2}}=x_{, \sigma}^{k} u^{\prime \prime \sigma}+\frac{\partial^{2} x^{k}}{\partial u^{\alpha} \partial u^{\beta}} u^{\prime \alpha} u^{\prime \beta},
$$

the Gauss equations ${ }^{9}$

$$
\frac{\partial^{2} x^{k}}{\partial u^{\alpha} \partial u^{\beta}}=\Gamma_{\alpha \beta}^{\gamma} x_{, \gamma}^{k}+d_{\alpha \beta} X^{k},
$$

and equations (2.5), equation (4.1) becomes

\footnotetext{
9 Eisenhart, loc. cit. pp. 215-216.
} 


$$
\epsilon_{i j k}\left(\bar{x}^{i}-x^{i}\right) x_{, \sigma}^{j} u^{\prime \sigma}\left(\rho^{\tau} x_{, \tau}^{k}+d_{\alpha \beta} u^{\prime \alpha} u^{\prime \beta} X^{k}\right)=0,
$$

where the $X^{k}$ are the components of the normal unit vector to $S$ at $P$, and the $d_{\alpha \beta}$ are the doubly covariant components of the second fundamental quadratic tensor of the surface. Since

$$
\epsilon_{i j k} x_{, \sigma}^{j} x_{, \tau}^{k}=\epsilon_{i j k} x_{, 1}^{j} x_{, 2}^{k} e_{\sigma \tau},
$$

and by use of (2.9), equation (4.4) may be written

$$
\epsilon_{i j k}\left(\bar{x}^{i}-x^{i}\right)\left[\frac{x_{, 1}^{j} x_{, 2}^{k}}{g^{1 / 2}} \Omega+x_{,{ }_{S}}^{i} X^{k} K_{n} u^{\prime \sigma}\right]=0,
$$

where the normal curvature $K_{n}$ is defined by

$$
K_{n} \equiv d_{\alpha \beta} u^{\prime \alpha} u^{\prime \beta} \text {. }
$$

Differentiation with respect to each of the parameters $u^{\prime 1}$ and $u^{\prime 2}$ gives the two equations

$$
\begin{array}{r}
\epsilon_{i j k}\left(\bar{x}^{i}-x^{i}\right)\left[\frac{x, 1 x^{j}, 2}{g^{1 / 2}} \frac{\partial \Omega}{\partial u^{\prime \tau}}+x_{, \sigma}^{j} X^{k}\left\{K_{n} \frac{\partial u^{\prime \sigma}}{\partial u^{\prime \tau}}+u^{\prime \sigma} \frac{\partial K_{n}}{\partial u^{\prime \tau}}\right\}\right]=0 \\
(\tau=1,2) .
\end{array}
$$

Since $K_{n} u^{\prime \sigma}$ and $\Omega$ are homogeneous of degree three in $u^{\prime 1}$ and $u^{\prime 2}$, Euler's relation for homogeneous functions shows that the three planes given by (4.6) and (4.8) are coaxial. Consequently, the characteristic lines of the envelope of the one-parameter family of osculating planes (4.6) are the intersections of the planes given by (4.8).

The direction numbers of the normals to the planes (4.8) are

$$
\begin{array}{rlr}
a_{m} \equiv & \frac{\partial \Omega}{\partial u^{\prime 1}} X^{m}+\left(K_{n}+u^{\prime 1} \frac{\partial K_{n}}{\partial u^{\prime 1}}\right) \epsilon_{m j k} x_{, 1}^{j} X^{k} & \\
& +\left(u^{\prime 2} \frac{\partial K_{n}}{\partial u^{\prime 1}}\right) \epsilon_{m j k} x_{, 2}^{j} X^{k} \quad & \\
b_{n} \equiv & \frac{\partial \Omega}{\partial u^{\prime 2}} X^{n}+\left(u^{\prime 1} \frac{\partial K_{n}}{\partial u^{\prime 2}}\right) \epsilon_{n j k} x_{, 1}^{j} X^{k} \\
& +\left(K_{n}+u^{\prime 2} \frac{\partial K_{n}}{\partial u^{\prime 2}}\right) \epsilon_{n j k} x_{, 2}^{j} X^{k} &
\end{array}
$$

since $\epsilon_{i j k} x_{, 1}^{j} x_{, 2}^{k} / g^{1 / 2}=X^{i}$. The direction numbers $c^{h}$ of the intersection of the two planes (4.8) are $c^{h} \equiv \epsilon^{h m n} a_{m} b_{n}$, which by neglecting zero de- 
terminants, interchanging columns, and renaming dummy indices when desirable, become

$$
\begin{aligned}
c^{h} \equiv \epsilon^{h m n} & {\left[\left\{\frac{\partial \Omega}{\partial u^{\prime 1}}\left(u^{\prime 1} \frac{\partial K_{n}}{\partial u^{\prime 2}}\right)-\frac{\partial \Omega}{\partial u^{\prime 2}}\left(K_{n}+u^{\prime 1} \frac{\partial K_{n}}{\partial u^{\prime 1}}\right)\right\} X^{m} \epsilon_{n j k} x_{, 1}^{j} X^{k}\right.} \\
+ & \left\{\frac{\partial \Omega}{\partial u^{\prime 1}}\left(K_{n}+u^{\prime 2} \frac{\partial K_{n}}{\partial u^{\prime 2}}\right)-\frac{\partial \Omega}{\partial u^{\prime 2}}\left(u^{\prime 2} \frac{\partial K_{n}}{\partial u^{\prime 1}}\right)\right\} X^{m} \epsilon_{n j k} x_{, 2}^{j} X^{k} \\
+ & \left\{\left(K_{n}+u^{\prime 1} \frac{\partial K_{n}}{\partial u^{\prime 1}}\right)\left(K_{n}+u^{\prime 2} \frac{\partial K_{n}}{\partial u^{\prime 2}}\right)\right. \\
& \left.\left.-\left(u^{\prime 1} \frac{\partial K_{n}}{\partial u^{\prime 2}}\right)\left(u^{\prime 2} \frac{\partial K_{n}}{\partial u^{\prime 1}}\right)\right\} \epsilon_{m j k} x_{, 1}^{j} X^{k} \epsilon_{n \epsilon_{s}} x_{, 2}^{r} X^{s}\right] .
\end{aligned}
$$

Using the facts $\epsilon_{i j k} x,{ }_{1}^{i} x,{ }_{2}^{j} X^{k}=g^{1 / 2}, X^{i} X^{i}=1, x,{ }_{\alpha}^{i} X^{i}=0$, and a well known formula for the simplification of a triple cross product of vectors gives

$$
\begin{aligned}
\epsilon^{h m n} X^{m} \epsilon_{n j k} x_{, 1}^{j} X^{k} & =x_{, 1}^{h}, \\
\epsilon^{h m n} X^{m} \epsilon_{n j k} x_{, 2}^{j} X^{k} & =x_{, 2}, \\
\epsilon^{h m n} \epsilon_{m j k} x_{, 1}^{j} X^{k} \epsilon_{n r s} x_{, 2}^{r} X^{s} & =g^{1 / 2} X^{h} .
\end{aligned}
$$

Euler's formulas for the homogeneous functions $K_{n}$ and $\Omega$ are

$$
u^{\prime 1} \frac{\partial K_{n}}{\partial u^{\prime 1}}+u^{\prime 2} \frac{\partial K_{n}}{\partial u^{\prime 2}}=2 K_{n}, \quad u^{\prime 1} \frac{\partial \Omega}{\partial u^{\prime 1}}+u^{\prime 2} \frac{\partial \Omega}{\partial u^{\prime 2}}=3 \Omega
$$

Use of (4.9) and (4.10) reduces the expression for $c^{h}$ to the simple form

$$
\begin{aligned}
c^{h} \equiv & 3\left(\Omega \frac{\partial K_{n}}{\partial u^{\prime 2}}-K_{n} \frac{\partial \Omega}{\partial u^{\prime 2}}\right) x_{, 1}^{h}+3\left(K_{n} \frac{\partial \Omega}{\partial u^{\prime 1}}-\Omega \frac{\partial K_{n}}{\partial u^{\prime 1}}\right) x_{, 2}^{h} \\
& +3 g^{1 / 2}\left(K_{n}\right)^{2} X^{h}
\end{aligned}
$$

giving $c^{h}$ as a single-valued function of the point $\left(u^{1}, u^{2}\right)$ and the direction $u^{\prime 1}, u^{\prime 2}$, except at points and directions on the surface for which both $K_{n}=0$ and $\Omega=0$. In this case there is no unique plane (4.6) which has contact of the second order with the hypergeodesic in the direction $u^{\prime \alpha}$ for which $K_{n}=0$ and $\Omega=0$; that is, all the planes containing the tangent line to this hypergeodesic will have contact of the second order with it. Consequently, the tangent line may be considered an element of the cone and in an asymptotic direction since $K_{n}=0$. 
At a point $\left(u^{1}, u^{2}\right)$ and in a direction $u^{\prime 1}, u^{\prime 2}$ such that $K_{n}=0$ and $\Omega \neq 0$, (4.11) has the form

$$
c^{h}=3 \Omega g^{1 / 2} \epsilon^{\alpha \beta} \frac{\partial K_{n}}{\partial u^{\prime \beta}} x_{, \alpha}^{h} .
$$

Now the vectors $u^{\prime \alpha}$ and $\epsilon^{\alpha \beta} \partial K_{n} / \partial u^{\prime \beta}$ are in the same direction, as is evident from the fact that the sine of the angle between the two vectors is zero since the cross product

$$
\epsilon_{\alpha \gamma} \epsilon^{\alpha \beta} \frac{\partial K_{n}}{\partial u^{\prime \beta}} u^{\prime \gamma}=\delta_{\gamma}^{\beta} \frac{\partial K_{n}}{\partial u^{\prime \beta}} u^{\prime \gamma}=\frac{\partial K_{n}}{\partial u^{\prime \gamma}} u^{\prime \gamma}=2 K_{n}=0 .
$$

Therefore, also in this case the element of the cone is tangent to the surface in an asymptotic direction.

At a point $\left(u^{1}, u^{2}\right)$ and in a direction $u^{\prime 1}, u^{\prime 2}$ such that $K_{n} \neq 0,(4.11)$ may be written

$$
c^{h}=3 g^{1 / 2}\left(K_{n}\right)^{2}\left[\epsilon^{\beta \alpha} \frac{\partial}{\partial u^{\prime \beta}}\left(\frac{\Omega}{K_{n}}\right) x_{, \alpha}^{h}+X^{h}\right] .
$$

If we discard the nonzero multipliers, the expressions for $c^{h}$ in (4.12) and (4.13) are replaced by

$$
c^{h}=\epsilon^{\alpha \beta} \frac{\partial K_{n}}{\partial u^{\prime \beta}} x^{h}, \quad\left(K_{n}=0, \Omega \neq 0\right)
$$

and

$$
c^{h}=r^{\alpha} x_{\alpha}^{h}+X^{h} \quad\left(K_{n} \neq 0\right),
$$

where

$$
r^{\alpha} \equiv \epsilon^{\beta \alpha} \frac{\partial}{\partial u^{\prime \beta}}\left(\frac{\Omega}{K_{n}}\right)
$$

defines the contravariant components of a vector which is the projection of $c^{h}$ onto the tangent plane to the surface. The assumption that the surface be expressible with the asymptotic curves as a coordinate net insures that in (4.14) $\partial K_{n} / \partial u^{\prime \beta}=0$ for both $\beta=1$ and $\beta=2$ is impossible; so (4.14) will have meaning.

5. A geometric interpretation of hypergeodesic curvature. It will be shown that the hypergeodesic curvature of a curve $C, u^{\alpha}=u^{\alpha}(s)$, is the curvature of the curve $C^{\prime}$ which is the projection of $C$ upon the tangent plane at the point $P$, the lines of projection being parallel to that element of the cone determined by the direction $u^{\prime \alpha}$ at $P$. 
Let $S^{\prime}$ denote the cylindrical surface consisting of the parallel lines of projection. If $1 / R$ is the normal curvature of $S^{\prime}$ in the direction of $C$ at $P$, and $\alpha$ is the angle between the principal normal to $C$ and the normal to $S^{\prime}$ at $P$, then by the theorem of Meusnier

$$
e / R=\rho^{-1} \cos \alpha,
$$

where $e= \pm 1$, and $\rho^{-1}$ is the curvature of $C$ at $P$. Also, if $1 / r$ is the curvature of $C^{\prime}$ at $P$, and if $\beta$ is the angle between the principal normal to $C^{\prime}$ and the normal to $S^{\prime}$ at $P$, then

$$
e / R=r^{-1} \cos \beta \text {. }
$$

After eliminating $R$ from these two equations, it is found that

$$
e / r=\frac{\cos \alpha}{\rho \cos \beta} .
$$

Computation of $\cos \alpha$ and $\cos \beta$ yields the formulas

$$
\cos \alpha=\frac{-\rho}{L \sin \phi}\left[K_{0}-K_{n} \epsilon_{\gamma \delta} u^{\prime} \gamma^{\delta} \delta\right] \quad\left(K_{n} \neq 0\right)
$$

and

$$
\cos \beta=\frac{-1}{L \sin \phi}
$$

where $\phi$ is the angle between the vector $c^{i}$, as given by (4.15), and the unit tangent vector $x,_{\sigma}^{\prime} u^{\prime \sigma}$ to the curves $C$ and $C^{\prime}$, and $L$ is the length of the vector $c^{i}$. Now, by (4.16) and the fact that $\Omega / K_{n}$ is homogeneous of degree one in the parameters $u^{\prime \alpha}$, it can be shown that

$$
\epsilon_{\gamma \delta} u^{\prime} \gamma_{\gamma} \delta=\Omega / K_{n} \quad\left(K_{n} \neq 0\right) .
$$

Substitution of the formulas for $\cos \alpha$ and $\cos \beta$ into (5.3) with use of (5.6) gives

$$
e / r=K_{o}-\Omega \equiv K_{h} \quad\left(K_{n} \neq 0\right),
$$

that is, the curvature of the curve $C^{\prime}$ is exactly the hypergeodesic curvature of the curve $C$ whenever the curve $C$ is not in an asymptotic direction. When the curve $C$ is in an asymptotic direction at $P$, the element of the cone determining the direction of the required projection of $C$ will lie in the tangent plane, and consequently will not give a proper projection of $C$ onto the tangent plane.

6. Hypergeodesic torsion of a curve. The torsion at $P$ of the hyper- 
geodesic $u^{\alpha}=u^{\alpha}(s)$ having the same direction as the curve $C$ at $P$ will be called the hypergeodesic torsion $\tau_{h}$ of $C$ at the point $P$.

Let $\alpha^{i}, \beta^{i}, \gamma^{i}$, be the tangent, principal normal, and binormal unit vectors of the hypergeodesic in the direction of $C$ at $P$. Since the principal normal $\beta^{i}$ of this hypergeodesic lies in the plane of $\alpha^{i}$ and $c^{i}$, the element of the cone given by (4.15), it follows that

$$
\beta^{i}=a \alpha^{i}+b c^{i}
$$

where $a$ and $b$ are to be determined. Multiplying (6.1) by $\alpha^{i}$ and summing gives

$$
a=-b c^{i} \alpha^{i}=-b L \cos \phi,
$$

and multiplying (6.1) by $\beta^{i}$ and summing gives

$$
1 / b=\beta^{i} c^{i}=L \sin \phi .
$$

Substitution of the values of $a$ and $b$ obtained from (6.2) and (6.3) into (6.1) yields

$$
\beta^{i}=\csc \phi\left[\frac{c^{i}}{L}-\cos \phi \alpha^{i}\right] .
$$

The restriction that $K_{n} \neq 0$ occurs in (6.4) since in general a hypergeodesic in an asymptotic direction has $c^{i}$ in coincidence with $\alpha^{i}$ making $\phi=0$ so that (6.4) would be meaningless.

From the Frenet-Serret formula $d \gamma^{i} / d s=\tau_{h} \beta^{i}$ multiplied by $\beta^{i}$ and summed arises

$$
\tau_{h}=\beta^{i} \frac{d \gamma^{i}}{d s}
$$

Now $\gamma^{i}$ is given by $\epsilon_{i j k} \alpha^{i} \beta^{k}$, which by $\alpha^{j}=d x^{j} / d s$ and (6.4) reduces to

$$
\gamma^{i}=\csc \phi \epsilon_{i j k} \frac{d x^{j}}{d s} \frac{c^{k}}{L} .
$$

Differentiation of (6.6) with respect to $s$ yields

$$
\begin{aligned}
\frac{d \gamma^{i}}{d s}= & -\csc \phi \cot \phi \frac{d \phi}{d s} \epsilon_{i j k} \frac{d x^{j}}{d s} \frac{c^{k}}{L}+\csc \phi \epsilon_{i j k} \frac{d^{2} x^{j}}{d s^{2}} \frac{c^{k}}{L} \\
& +\csc \phi \epsilon_{i j k} \frac{d x^{j}}{d s} \frac{d}{d s}\left[\frac{c^{k}}{L}\right] .
\end{aligned}
$$

By the substitution of the expressions for $\beta^{i}$ and $d \gamma^{i} / d s$, omission of 
the zero determinants, and a change of dummy indices, (6.5) becomes

$$
\tau_{h}=\csc ^{2} \phi \epsilon_{i j k} \frac{c^{i}}{L} \frac{d x^{j}}{d s}\left[\frac{d}{d s}\left(\frac{c^{k}}{L}\right)-\cos \phi \frac{d^{2} x}{d s^{2}}\right] .
$$

Since the components $c^{k} / L$ are scalars relative to transformations of surface coordinates, the partial derivatives of $c^{k} / L$ with respect to $u^{\alpha}$ are the same as the covariant derivatives of $c^{k} / L$ with respect to $u^{\alpha}$; therefore, the derivative of $c^{k} / L$ with respect to $s$ may be written

$$
\frac{d}{d s}\left(\frac{c^{k}}{L}\right)=\left(\frac{c^{k}}{L}\right),{ }_{\alpha} u^{\prime \alpha}+\frac{\partial}{\partial u^{\prime \gamma}}\left(\frac{c^{k}}{L}\right) u^{\prime \prime \gamma} .
$$

From (4.15) the covariant derivative of $c^{k} / L$ takes the form

$$
\left(\frac{c^{k}}{L}\right)_{, \alpha}=\frac{r^{\beta}}{L} x_{, \beta \alpha}^{k}+\frac{1}{L} X_{, \alpha}^{k}+\left(\frac{r^{\beta}}{L}\right)_{, \alpha} x_{, \beta}^{k}+\left(\frac{1}{L}\right)_{, \alpha} X^{k},
$$

which, by use of the Weingarten and Gauss equations ${ }^{10}$

$$
X_{, \alpha}^{k}=-d_{\alpha \gamma} g^{\gamma \beta} x_{, \beta}^{k}, \quad x_{, \alpha \beta}^{k}=d_{\alpha \beta} X^{k},
$$

may be written

$$
\left(\frac{c^{k}}{L}\right)_{, \alpha}=a_{\alpha}^{\beta} x_{\beta}^{k}+b_{\alpha} X^{k},
$$

where $a_{\alpha}^{\beta}$ and $b_{\alpha}$ are defined by

$$
\begin{aligned}
a_{\alpha}^{\beta} & \equiv\left(\frac{r^{\beta}}{L}\right)_{, \alpha}-\frac{1}{L} d_{\alpha \gamma} g^{\gamma \beta}, \\
b_{\alpha} & \equiv \frac{r^{\beta}}{L} d_{\alpha \beta}+\left(\frac{1}{L}\right), \alpha .
\end{aligned}
$$

Also, the partial derivatives of $c^{k} / L$ with respect to $u^{\prime \gamma}$ are given by

$$
\frac{\partial}{\partial u^{\prime} \gamma}\left(\frac{c^{k}}{L}\right)=\frac{\partial}{\partial u^{\prime \gamma}}\left(\frac{r^{\beta}}{L}\right) x_{, \beta}^{k}+\frac{\partial}{\partial u^{\prime \gamma}}\left(\frac{1}{L}\right) X^{k} \text {. }
$$

From (2.5), (4.2), and (4.3) it is evident that

$$
\frac{d^{2} x^{k}}{d s^{2}}=\rho^{\beta} x_{, \beta}^{k}+K_{n} X^{k}
$$

Hence, from the relations (6.9) through (6.13) it follows that

${ }^{10}$ Eisenhart, loc. cit. pp. 216-217. 


$$
\begin{aligned}
\frac{d}{d s}\left(\frac{c^{k}}{L}\right)-\cos \phi & \frac{d^{2} x^{k}}{d s^{2}} \\
= & \left\{\left[a_{\alpha}^{\beta}-\frac{r_{\alpha}}{L} \rho^{\beta}\right] x_{, \beta}^{k}+\left[b_{\alpha}-\frac{r_{\alpha}}{L} K_{n}\right] X^{k}\right\} u^{\prime \alpha} \\
& +\left\{\frac{\partial}{\partial u^{\prime \gamma}}\left(\frac{r^{\beta}}{L}\right) x_{, \beta}^{k}+\frac{\partial}{\partial u^{\prime \gamma}}\left(\frac{1}{L}\right) X^{k}\right\} u^{\prime \prime \gamma},
\end{aligned}
$$

in which use was made of the formula $\cos \phi=g_{\delta \alpha}\left(r^{\delta} / L\right) u^{\prime \alpha}$ $=\left(r_{\alpha} / L\right) u^{\prime \alpha}$.

Substitution of (6.14) in (6.8), if one uses (4.2) and (4.15) and neglects the zero determinants, gives

$$
\begin{aligned}
\tau_{h}= & \csc ^{2} \phi \epsilon_{i j k}\left\{\underset{x, \beta}{i} x_{, \delta}^{j} X^{k} \frac{r^{\beta}}{L} u^{\prime \delta}\left[b_{\alpha}-\frac{r_{\alpha}}{L} K_{n}\right] u^{\prime \alpha}\right. \\
& +X^{i} x_{, \delta}^{j} x_{, \beta}^{k}\left(\frac{1}{L}\right) u^{\prime \delta}\left[a_{\alpha}^{\beta}-\frac{r_{\alpha}}{L} \rho^{\beta}\right] u^{\prime \alpha} \\
& +x_{, \beta}^{i} x_{, \delta}^{j} X^{k} \frac{r^{\beta}}{L} u^{\prime \delta} \frac{\partial}{\partial u^{\prime \gamma}}\left(\frac{1}{L}\right) u^{\prime \prime \gamma} \\
& \left.+X^{i} x_{, \delta}^{j} x_{, \beta}^{k}\left(\frac{1}{L}\right) u^{\prime \delta} \frac{\partial}{\partial u^{\prime \gamma}}\left(\frac{r^{\beta}}{L}\right) u^{\prime \prime \gamma}\right\}
\end{aligned}
$$

which by the fact that $\epsilon_{i j k} x_{j}^{j}, x_{, \delta}^{\jmath} X^{k}=\epsilon_{\beta \delta}$ and by a rearrangement and simplification of terms becomes

$$
\begin{aligned}
\tau_{h}= & \frac{\csc ^{2} \phi}{L} \epsilon_{\beta \delta}\left\{r^{\beta} b_{\alpha}-a_{\alpha}^{\beta}+\frac{r_{\alpha}}{L}\left[\rho^{\beta}-r^{\beta} K_{n}\right]\right\} u^{\prime \alpha} u^{\prime 8} \\
& -\frac{\csc ^{2} \phi}{L^{2}} \epsilon_{\beta \delta} \frac{\partial}{\partial u^{\prime \gamma}}\left(r^{\beta}\right) u^{\prime \prime \gamma} u^{\prime \delta} .
\end{aligned}
$$

However, for a hypergeodesic of the family under consideration it follows from (3.4), (5.6), and (3.9) that

$$
\epsilon_{\beta \delta}\left[\rho^{\beta}-r^{\beta} K_{n}\right] u^{\prime \delta}=-K_{0}+\Omega=0 ;
$$

and from (4.16) it follows that

$$
\begin{aligned}
\epsilon_{\beta \delta} \frac{\partial}{\partial u^{\prime \gamma}}\left(r^{\beta}\right) u^{\prime \prime \gamma} u^{\prime \delta} & =\epsilon_{\beta \delta \epsilon^{\sigma \beta}} \frac{\partial^{2}}{\partial u^{\prime \gamma} \partial u^{\prime \sigma}}\left(\frac{\Omega}{K_{n}}\right) u^{\prime \prime \gamma} u^{\prime \delta} \\
& =-u^{\prime \delta} \frac{\partial}{\partial u^{\prime \delta}}\left[\frac{\partial}{\partial u^{\prime} \gamma}\left(\frac{\Omega}{K_{n}}\right) u^{\prime \prime \gamma}\right],
\end{aligned}
$$


or, since $\partial\left(\Omega / K_{n}\right) u^{\prime \prime \gamma} / \partial u^{\prime \gamma}$ is homogeneous of degree zero in the parameters $u^{\prime \alpha}$,

$$
\epsilon_{\beta \delta} \frac{\partial}{\partial u^{\prime \gamma}}\left(r^{\beta}\right) u^{\prime \prime} \gamma u^{\prime \delta}=0 .
$$

Therefore, (6.15) is reduced to

$$
\tau_{h}=\frac{\csc ^{2} \phi}{L} \epsilon_{\beta \delta}\left\{r^{\beta} b_{\alpha}-a_{\alpha}^{\beta}\right\} u^{\prime \alpha} u^{\prime \delta}
$$

which, by the definitions (6.11) of $a_{\alpha}^{\beta}$ and $b_{\alpha}$, may be written in the final form

$$
\begin{aligned}
\tau_{h}=\frac{\csc ^{2} \phi}{L} \epsilon_{\beta \delta}\left\{r ^ { \beta } \left[\left(\frac{1}{L}\right)_{, \alpha}+\right.\right. & \left.\frac{r^{\gamma}}{L} d_{\alpha \gamma}\right] \\
& \left.-\left[\left(\frac{r^{\beta}}{L}\right)_{, \alpha}-\frac{1}{L} d_{\alpha \gamma} g^{\gamma \beta}\right]\right\} u^{\prime \alpha} u^{\prime \delta} .
\end{aligned}
$$

The analytic expression (6.19) for the hypergeodesic torsion at $P$ of the curve $C$ with direction $u^{\prime \alpha}$, not an asymptotic direction, is a function of the point $P$, the direction $u^{\prime \alpha}$, and of course the particular family of hypergeodesics under consideration. Therefore, all curves $C$ with the same direction $u^{\prime \alpha}$ at $P$ will have the same hypergeodesic torsion at $P$ relative to the family under consideration.

7. A geometric condition that a hypergeodesic be a plane curve. In order to obtain a geometric condition equivalent to the condition that the torsion of a hypergeodesic be zero, namely that the expression (6.19) vanish, consider the developables of the complex of cone elements and their intersections with the surface. If only a very special set of developables is admitted, then the differential equation of the net of curves of intersection of these developables with the surface will be precisely the expression (6.19) set equal to zero. Hence, a hypergeodesic not in an asymptotic direction will be a plane curve if and only if it is a curve of this special related intersector net of the complex of cone elements of the family of hypergeodesics under consideration. The word net is used here in a more general sense to mean a set of curves consisting of two or more one-parameter families of curves.

To arrive at the differential equation of this special intersector net, a point with coordinates $x^{i}+t c^{i} / L$ is taken on the line of a cone element through $P$, and the point $P$ is allowed to move along a curve $C, u^{\alpha}=u^{\alpha}(s)$, for which the point $x^{i}+t c^{i} / L$ will describe a curve 
tangent to the line of the cone element through $P$. That is, $x^{i}+t c^{i} / L$ will generate the edge of regression of a developable. This developable will belong to the desired special set if $c^{i} / L$ at $P$ is taken as only that cone element which corresponds to the values $u^{\prime \alpha}$ for the curve $C$.

The above mentioned tangency to the cone element requires that

$$
d\left(x^{i}+t \frac{c^{i}}{L}\right)=d x^{i}+t d\left(\frac{c^{i}}{L}\right)+\frac{c^{i}}{L} d t=m \frac{c^{i}}{L},
$$

where $m$ is to be determined. Multiplication by $c^{i} / L$ and use of $\left(c^{i} / L\right)\left(c^{i} / L\right)=1$ and $\left(c^{i} / L\right) d\left(c^{i} / L\right)=0$ gives $m=d t+\left(c^{i} / L\right) d x^{i}$ so that (7.1) after a division by $d s$ becomes

$$
\frac{d x^{i}}{d s}-\frac{c^{i}}{L} \frac{c^{j}}{L} \frac{d x^{j}}{d s}+t \frac{d}{d s}\left(\frac{c^{i}}{L}\right)=0 .
$$

Replacing the derivatives with respect to $s$ by use of the relations (4.2) and (6.9) and multiplying by $\left(c^{i} / L\right)_{, \beta}$ yields

$$
\begin{aligned}
& {\left[\left(\frac{c^{i}}{L}\right)_{\beta} x_{, \alpha}^{i}-\left(\frac{c^{i}}{L}\right)_{, \beta} \frac{c^{i}}{L} \frac{c^{j}}{L} x_{, \alpha}^{j}\right] u^{\prime \alpha}} \\
& +\left[\left(\frac{c^{i}}{L}\right)_{, \beta}\left(\frac{c^{i}}{L}\right){ }_{\alpha} u^{\prime \alpha}+\left(\frac{c^{i}}{L}\right)_{\beta} \frac{\partial}{\partial u^{\prime \alpha}}\left(\frac{c^{i}}{L}\right) u^{\prime \prime \alpha}\right] t=0 .
\end{aligned}
$$

From (4.15) and (6.10) it follows that

$$
\begin{aligned}
\left(\frac{c^{i}}{L}\right)_{, \beta}^{i} x_{, \alpha}^{i} & =\left(a_{\beta}^{\delta} x_{, \delta}^{i}+b_{\beta} X^{i}\right) x_{, \alpha}^{i}=a_{\beta}^{\delta} g_{\delta \alpha}, \\
\frac{c^{i}}{L} x_{, \beta}^{i} & =\left(\frac{r^{\alpha}}{L} x_{, \alpha}^{i}+\frac{1}{L} X^{i}\right) x_{, \beta}^{i}=\frac{r^{\alpha}}{L} g_{\alpha \beta}, \\
\left(\frac{c^{i}}{L}\right)_{, \alpha}\left(\frac{c^{i}}{L}\right)_{, \beta} & =a_{\alpha}^{\sigma} a_{\beta}^{\tau} g_{\sigma \tau}+b_{\alpha} b_{\beta}, \\
\left(\frac{c^{i}}{L}\right)_{, \beta} \frac{\partial}{\partial u^{\prime \alpha}}\left(\frac{c^{i}}{L}\right) & =a_{\beta}^{\sigma} g_{\sigma \delta} \frac{\partial}{\partial u^{\prime \alpha}}\left(\frac{r^{\delta}}{L}\right)+b_{\beta} \frac{\partial}{\partial u^{\prime \alpha}}\left(\frac{1}{L}\right),
\end{aligned}
$$

and

$$
\frac{c^{i}}{L}\left(\frac{c^{i}}{L}\right)_{, \beta}=\frac{r^{\alpha}}{L} a_{\beta}^{\gamma} g_{\alpha \gamma}+\frac{1}{L} b_{\beta}=0,
$$

where the zero in (7.8) occurs since covariant differentiation of $\left(c^{i} / L\right)\left(c^{i} / L\right)=1$ gives $\left(c^{i} / L\right)\left(c^{i} / L\right)_{\beta}=0$. Therefore, (7.3) reduces to the two equations 


$$
\begin{aligned}
& \stackrel{\delta}{a_{\beta} g_{\delta \alpha} u^{\prime \alpha}}+\left[\left\{\begin{array}{c}
\sigma \\
a_{\alpha} a_{\beta}^{\tau} g_{\sigma \tau}
\end{array}+b_{\alpha} b_{\beta}\right\} u^{\prime \alpha}\right. \\
& \left.+\left\{a_{\beta}^{\sigma} g_{\sigma \delta} \frac{\partial}{\partial u^{\prime \alpha}}\left(\frac{r^{\delta}}{L}\right)+b_{\beta} \frac{\partial}{\partial u^{\prime \alpha}}\left(\frac{1}{L}\right)\right\} u^{\prime \alpha}\right] t=0 \\
& (\beta=1,2) .
\end{aligned}
$$

Eliminating $t$ from these two equations gives the determinantal equation

$$
\begin{aligned}
& e^{\beta \epsilon} a_{\beta}^{\delta} g_{\delta \alpha} u^{\prime \alpha}\left[\left\{a_{\gamma}^{\sigma} a_{\epsilon}^{\tau} g_{\sigma \tau}+b_{\gamma} b_{\epsilon}\right\} u^{\prime \gamma}\right. \\
& \left.+\left\{a_{\epsilon}^{\sigma} g_{\eta \sigma} \frac{\partial}{\partial u^{\prime \gamma}}\left(\frac{r^{\eta}}{L}\right)+b_{\epsilon} \frac{\partial}{\partial u^{\prime \gamma}}\left(\frac{1}{L}\right)\right\} u^{\prime \prime \gamma}\right]=0 .
\end{aligned}
$$

The part of (7.10) involving $u^{\prime \prime \gamma}$ may be written, after replacing $b_{\epsilon}$ by $-r^{\eta} a_{\epsilon}^{\sigma} g_{\eta \sigma}$ as obtained from (7.8), in the form

$$
e^{\beta \epsilon} a_{\beta}^{\delta} g_{\delta \alpha} a_{\epsilon}^{\sigma} g_{\eta \sigma}\left[\frac{\partial}{\partial u^{\prime \gamma}}\left(\frac{r^{\eta}}{L}\right)-r^{\eta} \frac{\partial}{\partial u^{\prime \gamma}}\left(\frac{1}{L}\right)\right] u^{\prime \alpha} u^{\prime \prime \gamma} .
$$

However,

$$
e^{\beta \epsilon} a_{\beta}^{\delta} a_{\epsilon}^{\sigma}=a e^{\delta \sigma}, \quad e^{\delta \sigma} g_{\delta \alpha} g_{\eta \sigma}=g e_{\alpha \eta},
$$

where $a \equiv e_{\gamma \tau} a_{1}^{\gamma} a_{2}^{\tau}$ and $g \equiv e^{\gamma \tau} g_{\gamma 1} g_{\tau 2}$; so that after some simplification (7.11) may be written

$$
\frac{a g}{L} e_{\alpha \eta} \frac{\partial r^{\eta}}{\partial u^{\prime \gamma}} u^{\prime \alpha} u^{\prime \prime} \gamma
$$

which vanishes by (6.17). Consequently, equation (7.10) is reduced to

$$
\left.e^{\beta \epsilon}{\stackrel{\delta}{a_{\beta}} g_{\delta \alpha}\left[a_{\gamma}^{\sigma} a_{\epsilon}^{\tau} g_{\sigma \tau}\right.}+b_{\gamma} b_{\epsilon}\right] u^{\prime \alpha} u^{\prime \gamma}=0,
$$

which by replacing $b_{\epsilon}$ as obtained from (7.8) and by use of (7.12) becomes

$$
a g e_{\alpha \sigma}\left[a_{\gamma}^{\sigma}-r^{\sigma} b_{\gamma}\right] u^{\prime \alpha} u^{\prime \gamma}=0
$$

If we discard the nonzero factors $a$ and $g^{1 / 2}$, and if we use the fact that $g^{1 / 2} e_{\alpha \sigma}=-g^{1 / 2} e_{\sigma \alpha}=-\epsilon_{\sigma \alpha}$, the differential equation of the special intersector net of the complex of cone elements takes the form

$$
\epsilon_{\sigma \alpha}\left[r^{\sigma} b_{\gamma}-a_{\gamma}^{\sigma}\right] u^{\prime \alpha} u^{\prime \gamma}=0 .
$$

When equation (7.13) is compared with the formula (6.18) it is apparent that a hypergeodesic not in an asymptotic direction is a plane 
curve if and only if it is a curve of the special intersector net of the complex of cone elements. ${ }^{11}$

This result may be stated in a purely geometric manner as follows: a hypergeodesic not in an asymptotic direction is a plane curve if and only if the one-parameter family of cone elements, which are the elements of contact of the osculating planes of the hypergeodesic with the cones, constitutes a developable.

The differential equation (7.13), after use of (6.11) and (4.16) and clearing of fractions, will in general be homogeneous of degree eight in the parameters $u^{\prime \alpha}$; consequently, the differential equation is of the first order and the eighth degree. Therefore, the special intersector net in general has the property that through any point on the surface pass eight curves of the net. However, there are many cases of families of hypergeodesics for which an equation of lower degree than eight is obtained. Such is the case when the family of hypergeodesics under consideration is a family of union curves.

8. Union curves. A family of union curves is a family of hypergeodesics for which the osculating planes of all curves of the family through a point on $S$ form an axial pencil. In this case the cone degenerates into a line of a congruence, one through each point of the surface and not in the tangent plane. If the direction cosines $\xi^{i}$ of the lines of congruence are

$$
\xi^{i}=p^{\sigma} x^{i}, q X^{i}
$$

with $p^{\sigma}$ and $q$ as functions of $u^{1}$ and $u^{2}$ alone, then from (4.15) it follows that for a family of union curves

$$
r^{\sigma}=\frac{p^{\sigma}}{q}, \quad \frac{r^{\sigma}}{L}=p^{\sigma}, \quad \frac{1}{L}=q,
$$

so that, by (5.6), $\Omega$ must have the form

$$
\Omega=K_{n} \epsilon_{\alpha \sigma} u^{\prime \alpha} \frac{p^{\sigma}}{q} .
$$

It is apparent that for a family of union curves the factor $K_{n}$ may be discarded from equation (4.6) of the osculating planes, since $\Omega$ has $K_{n}$ as a factor. Therefore, for a union curve in an asymptotic direction equation (4.6) can be considered to define a unique osculating plane which will contain the line $\xi^{i}$ of the congruence. Hence, the difficulties

${ }^{11}$ A generalization of a corresponding theorem first proved for union curves by Sperry, loc. cit. p. 220. 
and exceptions which occur in the asymptotic directions for a general family of hypergeodesics are eliminated in the case of a family of union curves by taking (8.3) to define $\Omega$ even for $K_{n}=0$.

Now, (8.3) is stated as a theorem as follows: a necessary and suffcient condition that (3.9) be a differential equation of the family of union curves relative to the congruence $\xi^{i}$ is that the scalar $\Omega$ be given by (8.3). Substitution of the expression (4.7) for $K_{n}$ and the expression $\Omega_{\alpha \beta \gamma} u^{\prime \alpha} u^{\prime \beta} u^{\prime \gamma}$ for $\Omega$ in (8.3) yields a non-symmetric form for $\Omega_{\alpha \beta \gamma}$, namely,

$$
\Omega_{\alpha \beta \gamma}=\epsilon_{\alpha \sigma} \frac{p^{\sigma}}{q} d_{\beta \gamma} .
$$

When the value of $\Omega$ in (8.3) is substituted in the expression (3.8) for hypergeodesic curvature, the result is an expression for union curvature $K_{u}$ as found by Springer; namely, $K_{u}=K_{q}-K_{n} \epsilon_{\alpha \sigma} u^{\prime \alpha} p^{\sigma} / q$. Also, by use of (8.2), the expression (6.19) for hypergeodesic torsion reduces to the expression for union torsion $\tau_{u}$ as found by Springer; namely,

$$
\tau_{u}=\csc ^{2} \phi \epsilon_{\beta \delta}\left\{p^{\beta}\left[q, \alpha+p^{\gamma} d_{\alpha \gamma}\right]-q\left[p_{, \alpha}^{\beta}-q d_{\alpha \gamma} g^{\gamma \beta}\right]\right\} u^{\prime \alpha} u^{\prime \delta} .
$$

Likewise, the differential equation of the intersector net of the congruence $\xi^{i}$ obtained from (7.13) after a multiplication by $q$ and use of (6.11) and (8.2) is

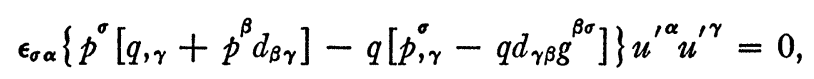

which is homogeneous of the second degree in the parameters $u^{\prime 1}$ and $u^{\prime 2}$. Consequently, in the case of a family of union curves the intersector net has only two curves through each point of the surface instead of eight. ${ }^{12}$

The geometric interpretation of union curvature is the same as that for hypergeodesic curvature with the one simplification that the method of projection (parallel to $\xi^{i}$ at $P$ ) is a function of the point $P$ only and not the direction $u^{\prime \alpha}$.

9. Geodesics. If the congruence $\xi^{i}$ for a family of union curves is normal to the surface, then the curves are the geodesic curves of the surface. The congruence is normal if and only if

$$
p^{\alpha} \equiv 0, \quad q \equiv 1 \quad(\alpha=1,2) .
$$

12 The curves of this net were originally called the torsal curves relative to the congruence by Sperry, and later referred to as $\Gamma$-curves by Lane and more recently as curves of the intersector net by Springer. 
From (8.3) it is apparent that the property (9.1) is equivalent to the condition that the scalar $\Omega$ be identically zero. As a result, the following theorem may be stated: a necessary and sufficient condition that a family of hypergeodesics defined by equation (3.9) be the family of geodesics is that the scalar $\Omega$ be identically zero in $u^{1}, u^{2}, u^{\prime 1}, u^{\prime 2}$, or what is equivalent, that the symmetric components $\Omega_{\alpha \beta \gamma}$ be identically zero in $u^{1}$ and $u^{2}$.

It is observed then from (3.8) that hypergeodesic curvature reduces to geodesic curvature when the family of hypergeodesics is the family of geodesics. Also, the geometric interpretation of hypergeodesic curvature reduces to the correct geometric interpretation for geodesic curvature, and the expression (6.19) for hypergeodesic torsion will reduce to the expression

$$
\tau_{\theta}=\epsilon^{\sigma \mu} d_{\alpha \sigma} \delta_{\delta \mu} u^{\prime \alpha} u^{\prime \delta}
$$

for geodesic torsion ${ }^{13} \tau_{g}$.

From the above discussion it is seen that hypergeodesic curvature and hypergeodesic torsion are proper generalizations of geodesic curvature and geodesic torsion since they are identical when the family of hypergeodesics under consideration is taken to be the family of geodesics.

UNIVERSITY OF WASHINGTON

${ }^{13}$ Eisenhart, loc. cit. p. 247. 